\title{
Performance of Distributed Energy Aware Routing (DEAR) Protocol with Cooperative Caching for Wireless Sensor Networks
}

\author{
Piyush Charan1, Tahsin Usmani' ${ }^{1}$, Rajeev Paulus ${ }^{2}$, Syed Hasan Saeed ${ }^{1}$ \\ ${ }^{1}$ Department of Electronics and Communication Engineering, Integral University, Lucknow, India \\ ${ }^{2}$ Department of Electronics and Communication Engineering, Sam Higginbottom University of Agriculture, Technology and \\ Sciences, Allahabad, India \\ Email: piyush@iul.ac.in
}

How to cite this paper: Charan, P., Usmani, T., Paulus, R. and Saeed, S.H. (2019) Performance of Distributed Energy Aware Routing (DEAR) Protocol with Cooperative Caching for Wireless Sensor Networks. Wireless Sensor Network, 11, 35-45. https://doi.org/10.4236/wsn.2019.113003

Received: February 10, 2019

Accepted: March 26, 2019

Published: March 29, 2019

Copyright $\odot 2019$ by author(s) and Scientific Research Publishing Inc. This work is licensed under the Creative Commons Attribution International License (CC BY 4.0).

http://creativecommons.org/licenses/by/4.0/

\begin{abstract}
Considering Wireless Sensor Networks (WSNs) in today's scenario, sending and receiving uninterrupted sensory data remains a challenge to achieve with minimal latency and energy consumption as low as possible. Energy consumption is exponentially growing in computing devices such as computers, embedded systems, portable devices, and wireless sensor networks. Extensive research has been in practice recently to minimize energy consumption without compromising the Quality of Service (QoS) that is to provide data to the requester node with minimum Delay and high Reliability. In this paper, a cooperative caching algorithm is used with the proposed Distributed Energy Aware Routing (DEAR) protocol that attempts to minimize energy consumption by reducing the packet overhead in the network and also providing the data to the requester with minimum delay by retrieving requested datum from the nearby caching node available in the vicinity of the requester or sink node. The simulation results clearly show that the energy consumption is less when the grid-based analytical model is used against the star/cluster based model while keeping the same necessary attributes.
\end{abstract}

\section{Keywords}

DEAR, WSNs, Energy Aware Routing, IEEE 802.15.4, ZigBee, Cooperative Caching

\section{Introduction}

ZigBee is a network and application layer specification based on the IEEE 802.15.4 MAC and PHY standard that is developed by the multi-vendor consortium, ZigBee Alliance. The ZigBee network layer supports the star, tree and mesh to- 
pologies. ZigBee based mesh networks are more suitable for practical applications, including home automation, intelligent transportation systems, personal healthcare, military communications, and so on. ZigBee device supports low data rate but exhibits enhanced energy consumption due to transceiving data packets, idle listening, overhearing, collisions and packet overheads due to multicast [1]. An efficient routing scheme is therefore essential in a mesh network so as to achieve efficient resources (e.g. Bandwidth and Energy Consumption). The possibility of achieving energy efficiency in such networks for AODV routing protocol has been explored previously by Charan et al. in [1]

In this paper, we analyze the proposed DEAR routing protocol with energy usage in ZigBee and present an improved solution for achieving better routing and energy efficiency by incorporating the localized caching algorithm that is the Caching in Cooperative Zones (CCZ) to overcome the limitation of AODV for energy efficiency usage. The rest of this paper is organized as follows. In Section 2 , the previous works related to the proposed scheme is discussed. Later in section 3 the proposed DEAR routing protocol in ZigBee is presented, and then our simulation model and parameters are discussed in Section 4. In Section 5, the various simulation results are presented and also we compare the simulation results of the DEAR_CCZ with the existing AODV_CCZ. Conclusions are drawn in the last section.

\section{Related Works}

Various cache related schemes have been proposed in the past until P. Charan et al. [1] proposed a caching scheme to be used in ZigBee based networks for AODV routing protocol. They had evaluated the performance of the proposed algorithm in two analytical models that are star based network model and the grid-based network model respectively. Previously, Jinbao Li et al. [2] proposed a caching scheme in a sensor network of multi-sink environment. In their approach, the network forms a set of network trees for a particular receiver. A common subtree is formed from these tree sets, and the root of the common subtree is selected as a data cache node to reduce communication costs.

J. Xu et al. [3] proposes a wait-caching scheme that expects data from the same cluster to become available within a threshold, then aggregates it with packets from the lower cluster and later sends it to the receiver, thereby reducing the number of packets transmitted. Md.A. Rahman et al. [4] proposed an efficient caching mechanism which negotiates data between the base station and the sensor, resulting in an expectation of data change and data loss.

T.P. Sharma et al. [5] proposed a cooperative caching scheme that used cooperation between various SNs in the defined area. In addition to its own local storage, nodes also use node storage from other clusters around them to form larger cache storage, called cumulative Cache. In this case, a token-based cache admission control (CAC) scheme is designed in which the node holding the token can cache or replace the data item. One of the disadvantages of the proposed model 
is that it generates a larger amount of packet overhead to maintain and rotate the token.

\section{Distributed Energy Aware Routing (DEAR) Protocol}

The DEAR protocol is an energy aware routing protocol that is an extension to the popular AODV routing protocol. The route discovery process in DEAR is different from AODV. The DEAR protocol is capable of generating routing paths based on the residual energy of nodes in the sensor network. DEAR calculates the routing path based on the Threshold value that is set in the protocol as $80 \%$ of the Average Energy of the Network. The Average energy of the network depends on the Total Link Cost that appears between two nodes " $\vec{i}$ " and " $"$ " while transmitting data from the source node " $i$ " to the destination node " $\vec{j}$ " as shown below in Figure 1.

The estimation of the Total Link Cost can be achieved in the following manner wherein, a sensor network based on the IEEE 802.15.4 standard is considered in which the traffic associated with the $i$-th mote be " $T_{i}$ " packets per second (pps). Let us assume that a data flow " $P$ " carries data traffic at a rate of " $t_{1}$ " pps, then $T_{i}$ can be estimated as:

$$
T_{i}=\sum_{l \in D(i)} t_{l}
$$

where, $D_{i} \rightarrow$ denotes the set of data flows through node " $i$.

The two important metrics for performance evaluation of a sensor network based on IEEE 802.15.4 are 1) Power Consumption, and 2) Quality of Services (QoS). Let $P_{i}(T)$ and $Q_{i}(T)$ be the power requirement and the Quality of Service requirements of a particular node " $\vec{i}$ " in the network when it carries $\mathrm{T}$ (pps).

If a new stream of data " $m$ " is added to node " $i$ " then this will result in a change in power consumption and QoS at that node.

Assuming that $p_{i}(T)$ be the instantaneous power consumption by the $i$-th

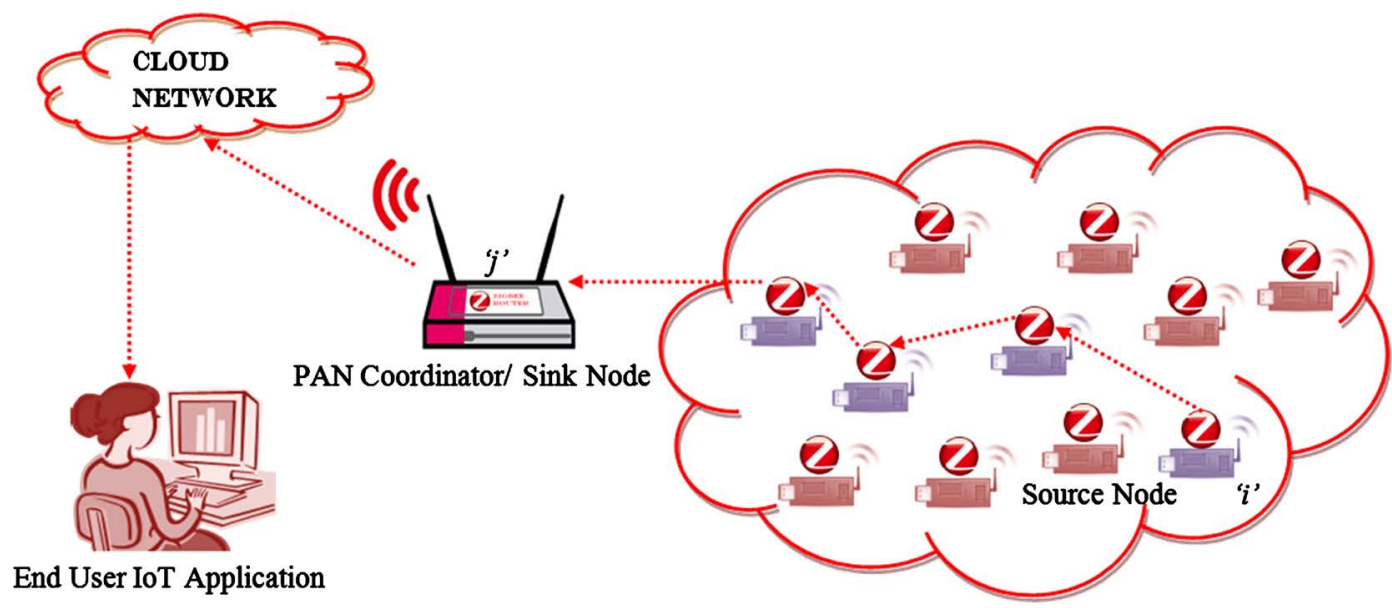

Figure 1. ZigBee based network in which data is transmitted from source node " $i$ " to destination or sink node " $"$ ". 
node when it carries " $T$ " packets per second that includes all aspects of packet processing: storing, routing and forwarding them through the neighboring nodes.

We now define the power cost asscoiated with the $m$-th data stream as $p_{i}^{m}\left(t_{m}, T_{i}\right)$ at node " $i$ " which represents the power consumption of the $m$-th flow in combination with the power associated with other flows. So,

$$
p_{i}^{m}\left(t_{m}, T_{i}\right)=a p_{i}\left(T_{i}+t_{m}\right)+b\left[p_{i}\left(T_{i}+t_{m}\right)-p_{i}\left(T_{i}\right)\right]
$$

where; $a, b \geq 0$. The first term is total power in $(\mathrm{mW})$ due to adding the $\mathrm{m}$-th flow, multiplied by some constant $a$; whereas, the second term represents the increase in energy for the other flows, multiplied by some constant $b$.

Hence the total power cost functions for the $m$-th data flow of rate " $t_{m}$ " on a routing path " $\lambda(i)$ " starting from node " $i$ " is written as:

$$
P_{\lambda(i)}^{m}\left(t_{m}, \overline{T_{\lambda(i)}}\right)=\sum_{n \in \lambda(i)} P_{n}^{m}\left(t_{m}, T_{n}\right)
$$

Similarly, the Quality of Service criterion, such as packet loss, delay or Packet Delivery Ratio (PDR) is given by

$$
Q_{\lambda(i)}^{m}\left(t_{m}, \overline{T_{\lambda(i)}}\right)=\sum_{n \in \lambda(i)} Q_{n}^{m}\left(t_{m}, T_{n}\right)
$$

where $\overline{T_{\lambda(i)}}=\left(T_{n_{1}}, T_{n_{2}}, \cdots, T_{n_{\lambda(\lambda)}}\right)$ where $n_{1}=i$ and $n_{j}$ are successive nodes in the routing path within the range $1 \leq j \leq|\lambda(i)|$.

Since, DEAR protocol is expected to minimize the overall cost of power consumption while satisfying the requested QoS, therefore, it is essential to optimize the total energy cost attribute, $T E C_{i}$ which will be a resultant of the power and the Quality of Service constraint. Hence, the total energy cost, $T E C_{i}$ will be represented as:

$$
T E C_{i}=P_{\lambda(i)}^{m}\left(t_{m}, \overline{T_{\lambda(i)}}\right)+\gamma Q_{\lambda(i)}^{m}\left(t_{m}, \overline{T_{\lambda(i)}}\right)
$$

where, $\gamma>0$ is a constant that matches the delay units with respect to power.

A sensor node will only be added to the routing path in a Distributed Energy Aware Routing (DEAR) protocol if the Threshold attribute is considered as a checkpoint. The node with $80 \%$ or more residual energy of the total energy cost will be included in a probable routing path. Thus, for a data to be sent from source node " $i$ " to the destination node " $j$ " the energy of the battery of nodes falling in its routing path must possess the minimum required threshold energy which is represented by

$$
\text { Threshold }=0.8 \times T_{E} C_{i j}
$$

The flowchart of the DEAR routing protocol is shown in Figure 2.

\section{Simulation Model and Parameters}

The proposed CCZ algorithm is simulated on NS-2 (version 2.32). The routing protocols used is DEAR for routing data traffic in a WSN based on the IEEE 802.15.4 PHY and MAC protocols and a free-space propagation model as a radio propagation model. The number of nodes in the network is 16 , and it is deployed in 


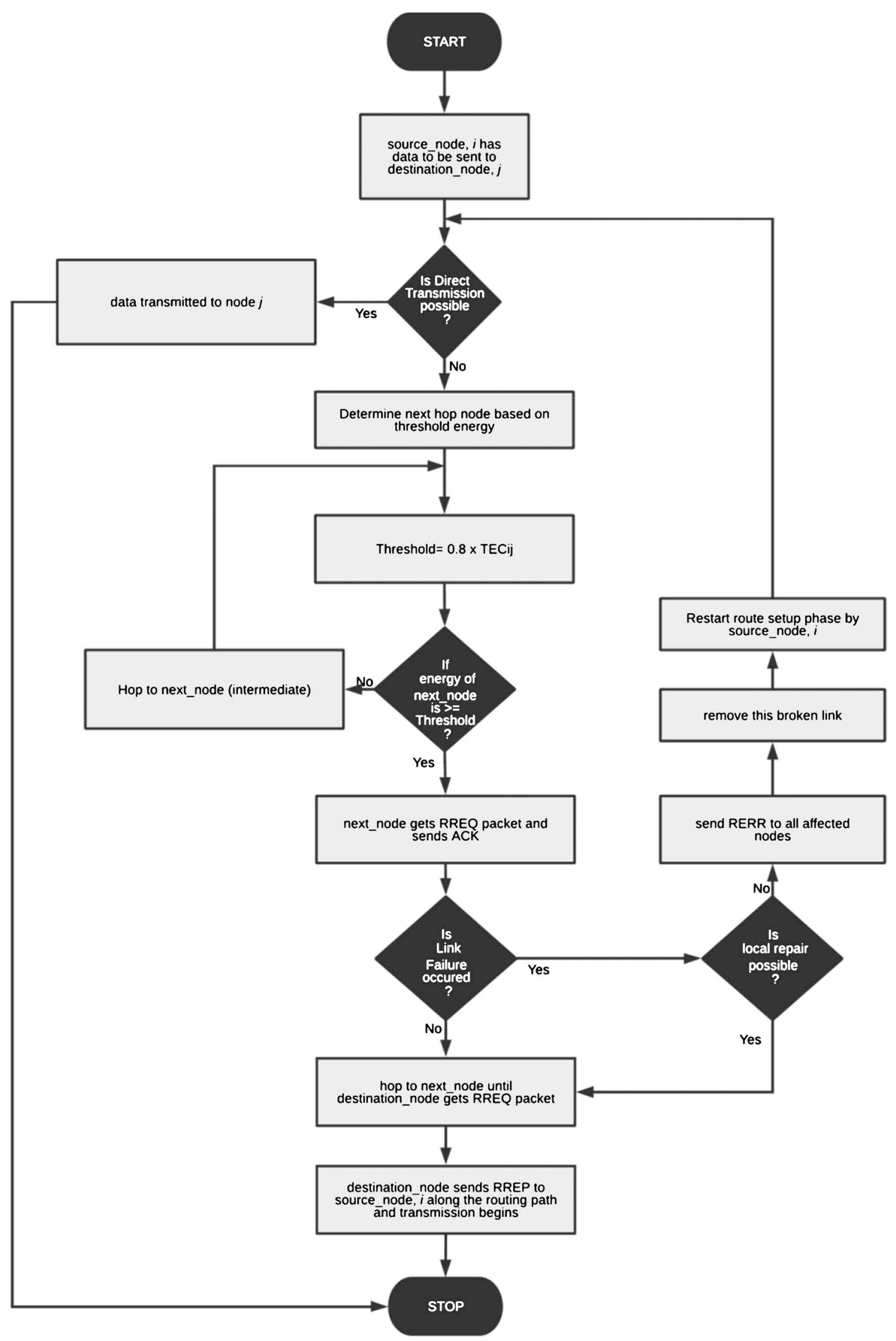

Figure 2. The flow diagram for DEAR protocol for WSN. 
a $100 \times 100$ square meter sensor area. The wireless bandwidth is $250 \mathrm{kbps}$, which represents the maximum amount of data or bits that can be transmitted over a period of time, typically one second. The other simulation parameters are illustrated in Table 1.

In Star (Cluster based) network consists of 16 sensor nodes based on IEEE 802.15.4 PHY and MAC standard are considered which are all Full Function Devices (FFDs) and are accompanied with a fixed amount of cache memory to store the sensed data as shown in Figure 3(a).

In Grid (peer-to-peer) based network, sixteen sensor nodes are placed equidistant in an area of $100 \mathrm{~m} \times 100 \mathrm{~m}$, and all devices are IEEE 802.15.4 PHY and MAC compliant, as shown in Figure 3(b). All devices are associated with some fixed amount of cache to store and update it with fresh data.

The IEEE 802.15.4 based sensor motes can generally be in one of the following modes: Idle, Transmit Mode, or Receive Mode. In the simulation we set the BO $=3$ and the $\mathrm{SO}=2$, for which the Duty Cycle comes out to be:

Duty cycle $=2^{-(\mathrm{BO}-\mathrm{SO})}=2^{-(3-2)}=2^{-1}=\frac{1}{2}=0.5$ or $50 \%$. A sensor node with very low duty cycle goes to sleep mode until a beacon signal arrives again to wake up the radio [6].

The following three performance metrics have been evaluated:

\section{1) Average Query Latency $\left(T_{q_{a v g}}\right)$}

The query latency " $T_{q}$ " can be defined as the time interval between a query sent by a requester and the response received back by the requester/sink. The average query latency is the query latency " $T_{q}$ " averaged over all the generated queries.

\section{2) Byte Hit Ratio (B)}

The byte hit ratio $B$ is defined as the ratio of total bytes of data retrieved from the cache to the total number of requested data bytes by the sink/requester node. The byte hit ratio $B$ includes bytes retrieved from a local hit known as a Local Byte Hit $\left(B_{\text {local }}\right)$; bytes retrieved from a zone hit known as a Zone Byte Hit $\left(B_{z o n e}\right)$ and the bytes retrieved from a remote hit known as a Remote Byte Hit $\left(B_{\text {remote }}\right)$. It is to be noted that bytes retrieved from a Global hit are freshly sensed data and

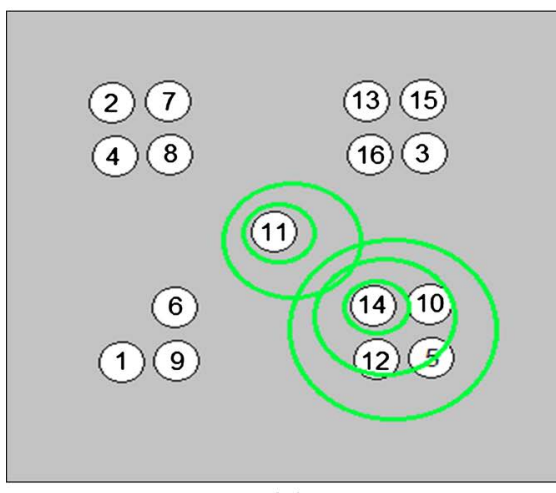

(a)

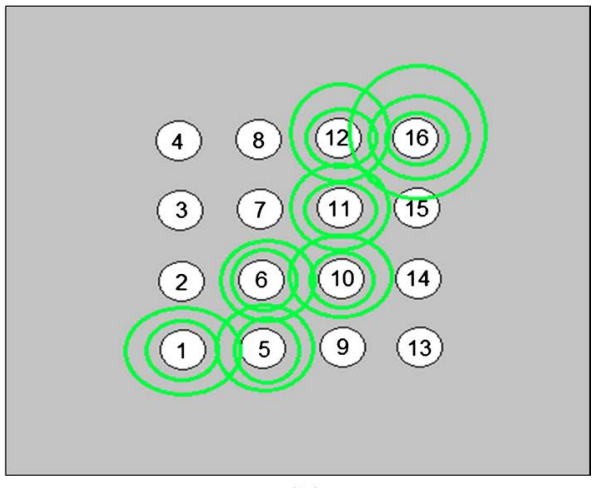

(b)

Figure 3. (a) Star based network model; (b) Grid based network model. 
Table 1. Simulation parameters for DEAR with cooperative caching.

\begin{tabular}{ccc}
\hline Parameter & Default Value & Range \\
\hline Number of Nodes & 16 & \\
Number of Data Items & 500 & \\
Payload Size & 64 bytes & \\
PHY and MAC Layer & IEEE 802.15 .4 & $100-300 \mathrm{~s}$ \\
Channel Frequency & $2.4 \mathrm{GHz}$ & $200-1400$ \\
Bandwidth (kbps) & 250 & \\
Waiting interval $\left(t_{w}\right)$ & $10 \mathrm{~s}$ & \\
TTL & $300 \mathrm{~s}$ & \\
Cache Size (KB) & 800 & \\
Traffic Type & CBR & \\
Routing Protocol & DEAR & \\
Beacon Order & 3 & 2 \\
Superframe Order &
\end{tabular}

The data items are updated at the source nodes. The source node serves the requests on First-Come-First-Serve (FCFS) Basis.

cannot be considered to have been retrieved from the cache.

\section{3) Total Energy Consumption $\left(E_{\text {total }}\right)$}

Total energy consumption is defined as the algebraic sum of energy consumed

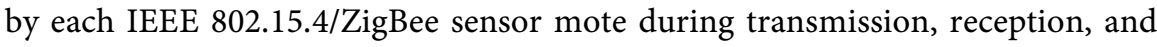
sleep modes. For simulation purpose, we consider the unit of energy consumption in $\mathrm{mWh}$.

$$
E_{\text {total }}=E_{\text {transmit }}+E_{\text {receive }}+E_{\text {sleep }}
$$

\section{Simulation Results}

Figure 4(a) shows the effect of varying cache sizes on the Average Query Latency for both the analytical network models that is Star/Cluster connected Network and the Grid Based Network. Simulation shows that the average query latency $\left(T_{q_{a v g}}\right)$ decreases as the size of the cache increases, since both cases satisfy more number of data queries. This is because as the size of the cache increases, more of the required data items can be found in the local cache. Average query latency in grid/peer-to-peer networking is less than in Star/Cluster based networks. Therefore, all of the CCZ caching algorithms in Grid Connected Network perform better than Star Connected Network.

Figure 4(b) shows the impact of varying cache size on byte hit ratio. Both the Star Connection Network and the Grid Connection Network show better byte hit rates as the size of the cache increases. When the cache size is small, the data is more contributed by zone hits and remote hits, but as the cache size is increased, the contribution of local hits becomes significant. This is because as the cache gets larger, a larger number of data items are found in the local cache. The 

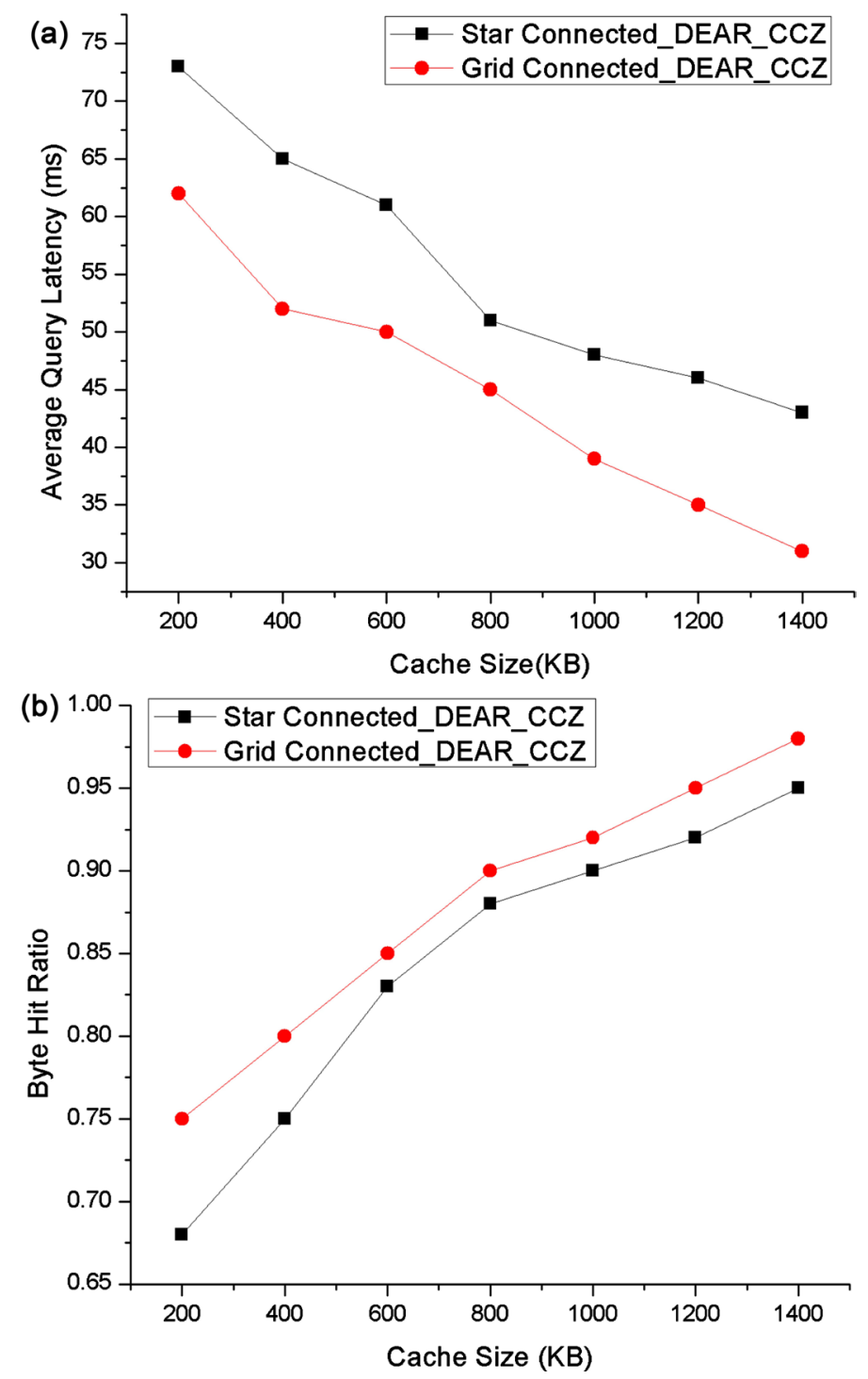

Figure 4. (a) Average query latency with different cache size for DEAR protocol; (b) Byte hit ratio with different cache size for DEAR protocol.

local byte hit rate increases as the size of the cache increases because more data can be cached locally for larger cache storage. According to the simulation results, the grid connection network has better byte hit ratio than the star / cluster connection network.

Figure 5(a) shows the total energy consumption of nodes in a network connected by a star (cluster) connection. Different data rates are considered in the range of 1 to 5 packets per second (pps). Two scenarios are considered in the analysis of energy consumption, in one case the DEAR routing protocol is considered and in the other case the DEAR routing protocol is used in cooperation with the $\mathrm{CCZ}$ cache algorithm in order to minimize the packet transmission delay and therefore the overall power consumption. Based on the results, we see that when the data rate is 5 pps, the node consumes less energy when combined with the caching algorithm; this is because the requester retrieves data from a 


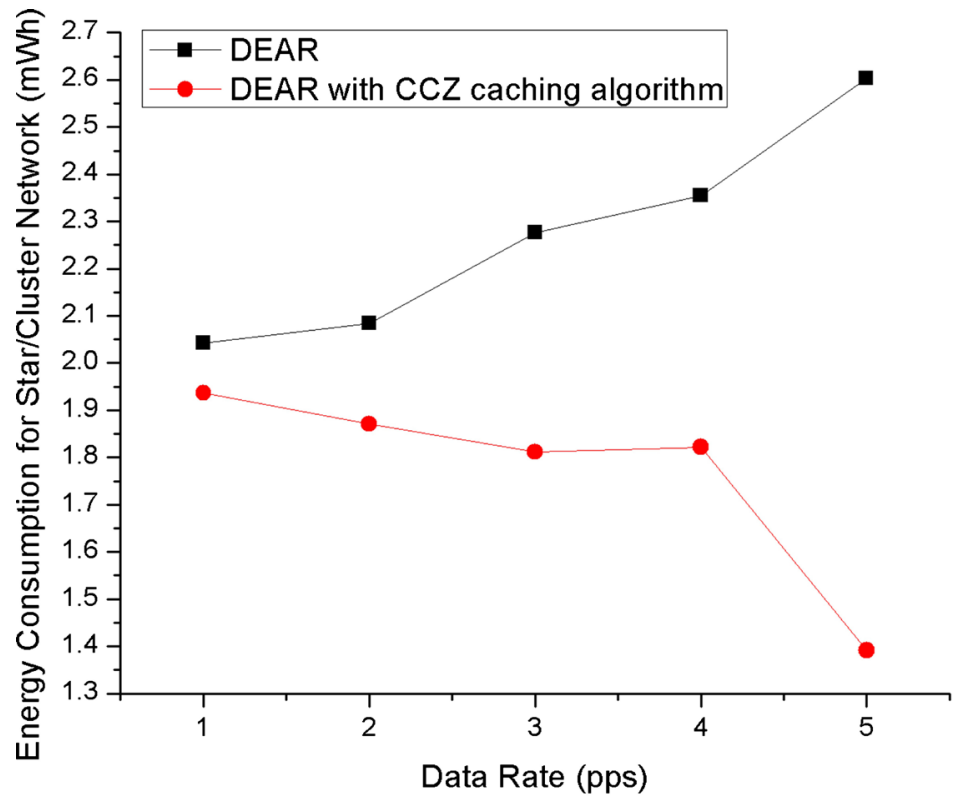

(a)

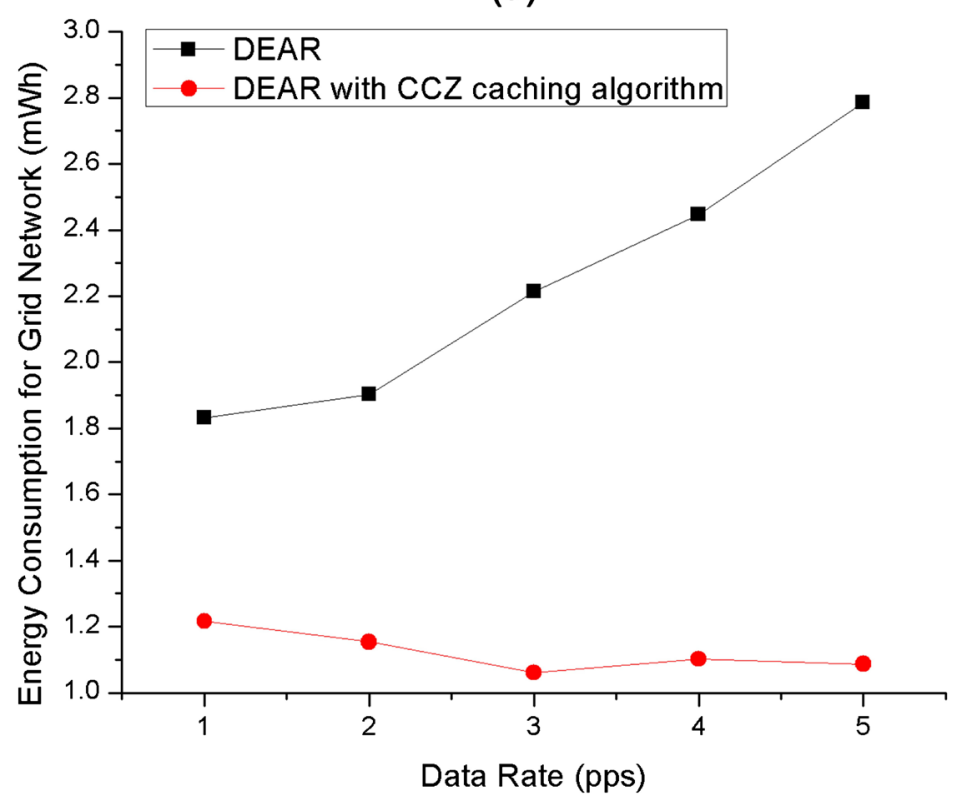

(b)

Figure 5. (a) Comparison of Energy Consumption in Star/Cluster Network for DEAR routing with and without CCZ caching algorithm; (b) Comparison of Energy Consumption in Grid/peer-to-peer Network for DEAR routing with and without CCZ caching algorithm.

nearby cache node or the local hit may have occurred, and the data item is retrieved from the requesting node itself. For the Star/Cluster network with DEAR routing protocol, the average node energy consumption is $2.27 \mathrm{mWh}$. However, when DEAR routing protocol and CCZ cache algorithm are used together, the average energy consumption is $1.77 \mathrm{mWh}$.

Figure 5(b) shows the total energy consumption of nodes when connected in a grid (peer-to-peer) connection. Different data rates are considered in the range of 1 to 5 packets per second (pps). The similar scenarios are considered while 
analyzing for energy consumption, that is one in which DEAR routing protocol is considered and in the other scenario, DEAR routing protocol is simulated with the CCZ Cooperative Caching Algorithm. The simulation results show that when only DEAR routing protocol is alone working then the energy consumption is increasing as the data rate is increased. However, when the CCZ caching algorithm comes into action with the DEAR routing protocol then the Energy Consumption continues to drop. For a Grid Network, the average energy consumption by nodes with only DEAR routing algorithm was $2.24 \mathrm{mWh}$. And when, DEAR routing protocol is used with the CCZ caching algorithm then the average energy consumption is $1.12 \mathrm{mWh}$.

Comparison of DEAR_CCZ with AODV_CCZ

\section{a) For Starl Cluster-based Network}

When AODV routing protocol is simulated with the localized CCZ caching algorithm then the average energy consumption was $2.43 \mathrm{mWh}$ for cluster network [1], whereas for the same network when DEAR routing protocol with CCZ algorithm is simulated then the energy consumption is $1.77 \mathrm{mWh}$. This result proves about the efficacy of the localized algorithm wherein the requester gets the requested datum from the nearby cache via the local hit, zone hit, remote hit or the global hit.

\section{b) For Gridl Peer-to-Peer based Network}

When AODV routing protocol is simulated with the localized CCZ caching algorithm then the average energy consumption was $1.92 \mathrm{mWh}$ for grid network [1], whereas for the same network when DEAR routing protocol with CCZ algorithm is simulated then the energy consumption is $1.12 \mathrm{mWh}$. This shows that the algorithm is serving the data to the requester by providing the data stored in the cache memory locally or remotely in a particular zone, thereby providing data to the source with minimal latency and higher accuracy.

\section{Conclusion}

Energy consumption by the nodes in the wireless environment is the cause of concern and minimization of Energy Consumption is the main crux of the proposed algorithm. The Simulation-based performance study was conducted to evaluate the proposed DEAR protocol with and without the use of localized caching algorithm; wherein the results show that the proposed scheme with the localized CCZ algorithm ensures that a query is served from the nearest cache or source for the network based on IEEE 802.15.4. Furthermore, the CCZ caching scheme performs significantly better in grid-based or peer-to-peer network model than the cluster based or star network model for DEAR routing protocol in terms of Byte Hit Ratio, Energy Consumption and the Average Query Latency. However, the future work would include a broader performance assessment for large ubiquitous network. We also intend to extend our scenario to a large heterogeneous wireless environment; wherein the devices of different wireless workgroup would be a part of the same network. 


\section{Acknowledgements}

We would like to thank Integral Information and Research Centre of Integral University, Lucknow, India for providing an opportunity to carry out this research work. This work is an intellectual property of Integral University vides the Manuscript Communication No. IU/R\&D/2019-MCN000600.

\section{Conflicts of Interest}

The authors declare no conflicts of interest regarding the publication of this paper.

\section{References}

[1] Charan, P., Usmani, T., Paulus, R. and Saeed, S.H. (2018) A Cooperative Cache Management Scheme for IEEE802.15.4 Based Wireless Sensor Networks. International Journal of Electrical and Computer Engineering, 8, 1701-1710. https://doi.org/10.11591/ijece.v8i3.pp1701-1710

[2] Li, J.B., Li, S.L. and Zhu, J.H. (2009) Data Caching Based Queries in Multi-Sink Sensor Networks. International Conference on Mobile Ad-hoc and Sensor Networks, Fujian, 14-16 December 2009, 9-16.

[3] Xu, J.F., Li, K.Q., Shen, Y.M. and Liu, J. (2008) An Energy-Efficient Waiting Caching Algorithm in Wireless Sensor Network. International Conference on Embedded and Ubiquitous Computing, Vol. 1, Shanghai, 17-20 December 2008, 323-329. https://doi.org/10.1109/EUC.2008.39

[4] Rahman, Md.A. and Hussain, S. (2007) Effective Caching in Wireless Sensor Network. International Conference on Advanced Information Networking and Applications Workshops, Vol. 1, Niagara Falls, 21-23 May 2007, 43-47.

[5] Sharma, T.P., Joshi, R.C. and Misra, M. (2008) Dual Radio Based Cooperative Caching for Wireless Sensor Networks. IEEE International Conference on Networking, New Delhi, 12-14 December 2008, 1-7.

https://doi.org/10.1109/ICON.2008.4772565

[6] Charan, P., Usmani, T., Paulus, R. and Saeed, S.H. (2016) Performance Evaluation of AODV Protocol for Energy Consumption and QoS in IEEE 802.15.4 Based Wireless Sensor Network Using QualNet Simulator. Wireless Sensor Network, 8, 166-175. https://doi.org/10.4236/wsn.2016.88014 